\title{
Co-Speech Gesture Mimicry in the Process of Collaborative Referring During Face-to-Face Dialogue
}

\author{
Judith Holler • Katie Wilkin
}

Published online: 23 January 2011

(C) Springer Science+Business Media, LLC 2011

\begin{abstract}
Mimicry has been observed regarding a range of nonverbal behaviors, but only recently have researchers started to investigate mimicry in co-speech gestures. These gestures are considered to be crucially different from other aspects of nonverbal behavior due to their tight link with speech. This study provides evidence of mimicry in co-speech gestures in face-to-face dialogue, the most common forum of everyday talk. In addition, it offers an analysis of the functions that mimicked co-speech gestures fulfill in the collaborative process of creating a mutually shared understanding of referring expressions. The implications bear on theories of gesture production, research on grounding, and the mechanisms underlying behavioral mimicry.
\end{abstract}

Keywords Co-speech gestures · Mimicry · Collaborative referring · Grounding · Common ground

\section{Introduction}

Much of everyday talk is accompanied by meaningful, spontaneous movements of the body, in particular the hands and arms. These 'co-speech gestures' are an integral part of human communication as they are tightly linked to speech semantically, pragmatically, and temporally; jointly, these two modalities are thus considered as constituting human language (Kendon 1980, 2000, 2004; McNeill 1985, 1992).

One central debate in the field of gesture studies has focused on the exact functions that co-speech gestures fulfill. Researchers have argued for communicative functions, with gestures benefiting primarily the addressee(s) (e.g., Bavelas 1994; Bavelas and Chovil

J. Holler · K. Wilkin

School of Psychological Sciences, University of Manchester, Coupland Building 1,

Oxford Road, Manchester M13 9PL, UK

J. Holler $(\bowtie)$

Max Planck Institute for Psycholinguistics, Wundtlaan 1,

6525 XD Nijmegen, The Netherlands

e-mail: judith.holler@mpi.nl 
2000; Clark 1996; Kendon 1983, 1985, 2004). Others have postulated that they fulfill predominantly cognitive functions benefiting the speaker him or herself, such as the facilitation of lexical access (e.g., Butterworth and Hadar 1989; Chawla and Krauss 1994; Morrel-Samuels and Krauss 1992; Pine et al. 2007) and conceptual planning (e.g., Hostetter et al. 2007; Kita and Davies 2009; Melinger and Kita 2007). However, these theories are not mutually exclusive (e.g., Alibali et al. 2001; Jacobs and Garnham 2007). Consequently, recent research has focused on exploring both of these types of functions and their underlying mechanisms in greater detail.

The present study forms part of this line of inquiry, with the aim to enhance our understanding of the communicative uses and functions of co-speech gestures. Past research has provided us with evidence that co-speech gestures contribute a significant amount of information to a speaker's message (e.g., Graham and Argyle 1975; Holler et al. 2009; Kelly and Church 1998). Moreover, this gestural information is often not contained in the speech at all.

However, evidence for co-speech gestures being communicative (in the sense that recipients seem to reliably glean information from them) does not necessarily mean that speakers actually produce them with communicative intent. In order to provide evidence that they do, paradigms tapping gesture production rather than comprehension have been employed. For example, corroborating evidence for gestures being communicatively intended comes from studies manipulating the social and communicative context of talk. Studies have observed effects of addressee location on gesture orientation and gesture space (Furuyama 2002; Özyürek 2002). Bavelas et al. (2002) showed that speakers describing stimuli to an imagined interlocutor, who they were told will either see a videorecording or hear a tape-recording of their description, produced more gestures when they thought their recording would be seen by the other person. Other studies have demonstrated similar effects during actual interaction comparing interlocutors' gestures when communicating face-to-face or with a visually occluded addressee (e.g., Alibali et al. 2001; Emmorey and Casey 2001; Gullberg 2006). In addition, Bavelas et al. (2008) recently found evidence for independent effects of visibility and dialogue on gesture use. Although speakers gestured slightly less when they were not able to see their addressee compared to face-to-face, the authors observed a significant drop-off in gesture rate only when speakers were talking into a tape recorder (i.e., producing pure monologue) instead of to a visually occluded but dialogically involved addressee. This supports the notion of co-speech gestures being communicatively intended as well as being an integral part of dialogue.

Apart from manipulations of overt, physical aspects of the communicative situation, research has also tapped into more covert processes, such as interactants' thinking and understanding. Holler and Beattie (2003) showed that speakers used more iconic gestures in association with lexically ambiguous speech (representing a potential problem for the addressee) than with non-ambiguous lexical items. In addition, researchers have manipulated the amount of interlocutors' mutually shared knowledge, or common ground (Clark 1996; Stalnaker 1978). For example, co-speech gestures have been found to be less precise in their form when interactants have more common ground (Gerwing and Bavelas 2004), speakers appear to use gestures at a lower rate when they are asked to tell the same story to the same recipient repeatedly (Jacobs and Garnham 2007), and gestures contain less information, at least when referring to the size of objects, when more common ground exists (Holler and Stevens 2007) (although this may be different for other types of semantic features, see Holler and Wilkin 2009).

Despite all this, what we know about the specific functions of co-speech gestures in face-to-face interaction is still sparse. Kendon $(1985,2004)$ provides a range of detailed 
descriptions illustrating different uses of gesture, such as the disambiguation of speech, the substitution of speech, as well as the emphasis and telescoping of information. Some equally interesting analyses have been provided by conversation analysts, identifying functions of gestures that impact on the sequential organization of conversation and the mutual orientation of those participating in it (e.g., de Fornel 1992; Goodwin 1986, 2000; Heath 1992; Streeck 1994). Further, Bavelas and her colleagues have identified a particular type of gesture, which they termed 'interactive gestures', as well as dialogue-specific functions associated with these concerned with the involvement of the conversational partner (Bavelas et al. 1992, 1995). However, the body of research in this area is still comparatively small, and a greater understanding of gestural uses and functions in social interaction is clearly needed.

One domain in which we lack knowledge in particular is the joint activity of creating a mutually shared understanding of what is being communicated. Clark and colleagues (e.g., Clark 1996; Clark and Brennan 1991; Clark and Schaefer 1987, 1989; Clark and WilkesGibbs 1986) made the important argument that language use cannot be conceptualized as two people being engaged in an activity consisting of separate components (i.e., speaking and listening) that can be studied in isolation. Rather, they argue that this process is inherently collaborative. This means that interactants create meaning jointly, through what has been referred to as an iterative process, with the aim of establishing mutual understanding (Clark and Wilkes-Gibbs 1986). For example, the collaborative creation of a definite reference involves the presentation of a noun phrase by A, as well as a response from B (such as signaling acceptance of A's noun phrase) in order to reach the mutual agreement that what has been said has been understood by both parties (Clark and WilkesGibbs 1986). This collaborative process has been referred to as grounding (Clark and Brennan 1991). As Clark and Brennan maintain, understanding is hardly ever perfect. They therefore claim that what interactants aim for with the process of grounding is to do as well as possible under given circumstances; "the contributor and his or her partners mutually believe that the partners have understood what the contributor meant to a criterion sufficient for current purposes. This is called the grounding criterion." (Clark and Brennan 1991, p. 129; original emphasis). Thus, the process of grounding is an incremental process, which requires constant and careful coordination of the individuals involved. This incremental, iterative process provides interactants with the basis for updating their common ground on a moment-by-moment basis (Clark and Brennan 1991).

Although we have some knowledge about how co-speech gesture is affected by mutually shared knowledge that exists from the outset of a conversation (such as through the shared experience of watching a video stimulus or acting on certain objects, as seen above), very little is known about their role in the interactive process that helps to establish it. A crucial question therefore is what kind of role, if any, co-speech gestures play during the collaborative process of language use, in particular with respect to the process of grounding. The present study is an attempt to provide some first insights in this respect. More specifically, the focus is on the phenomenon of co-speech gesture mimicry, which, we will argue, plays a significant role in this process.

Some recent studies have already begun to address the topic of mimicry in co-speech gesture, with the aim of showing that this phenomenon does exist (Kimbara 2006, 2008; Parrill and Kimbara 2006); of course, it has long been known that interactants mimic each other's nonverbal behaviors, such as posture (LaFrance 1979, 1985), foot shaking (Chartrand and Bargh 1999; Lakin et al. 2008), face touching and facial expressions (Chartrand and Bargh 1999; Lakin and Chartrand 2003; see Chartrand et al. 2005 for an overview). However, demonstrating that mimicry can also be observed in co-speech 
gestures was a significant step, as this modality is usually considered a fundamentally different form of nonverbal behavior-one that is closely tied to speech and involved in the communication of semantic meaning (Kendon 2000; McNeill 1992) rather than emotions and attitudes, which other nonverbal behaviors are believed to convey (Graham and Argyle 1975; Martin and Bateson 1994). What we have learned from these studies is that participants who observe gestural mimicry in a video-recorded interaction are more likely to also mimic individual features of these gestures in an interaction with another person (Parrill and Kimbara 2006). Kimbara (2008) built on this research by showing that mimicry also occurs in live interaction. In her study, two participants, sitting sideby-side, jointly narrated a cartoon story to a video camera with the experimenter located behind it as the recipient. In a control condition, the same joint narration took place but the two narrators were separated by an opaque screen while talking. The results showed that participants who were not separated, and hence were able to see each other's gestures, displayed more gestural mimicry than participants who were visually separated.

The present study advances this previous work by analyzing co-speech gesture mimicry in face-to-face dialogue, the most common forum of everyday discourse (Bavelas et al. 1992). While Kimbara's (2008) study took a very important step as it involved live interaction and mutual visibility between participants, these conversations were crucially different from face-to-face dialogue. The investigation focused on triadic communication (i.e., involving two narrators and one recipient) in the context of a rather specific conversational set-up; the two narrators were able to glance over to the other sitting next to them, but the actual transmission of information was from the narrators to a third person, in this case a passive recipient (with pre-existing knowledge of the story as this person was the experimenter). In this situation where co-narrators were required to provide a joint narrative for a third person, one of their major goals will have been to provide a coherent story allowing the recipient to easily match up the individual narrative parts coming from the different narrators. This is a form of talk where convergence (in both speech and gesture) is of utmost importance to successfully inform a third; in other words, displaying very similar behavior was here crucial.

Therefore, the question remains to what extent participants mimic each other's gestures in face-to-face dialogue, where one person informs the respective other without separate narrative components having to be merged for a third. The general goal in faceto-face dialogue is to convey meaning about entities, usually absent, requiring the interactants to work together in order to figure out whether they are talking about the same thing. That is, dialogue is based on the very principle of collaborative grounding with the aim to create mutually shared understanding. What we do have is individual examples of gesture mimicry occurring in spontaneous dyadic interaction (Kimbara 2006; Tabensky 2001), but no systematic analysis across a larger sample, and, more importantly, no evidence that these cases did not just occur by chance. The present study aims to close this gap.

The data presented here were obtained through an experimental paradigm based on Clark and Wilkes-Gibbs (1986) involving a referential communication task. In addition to their original experimental condition in which participants were separated by a wooden screen we introduced a face-to-face condition. The task required two people to engage in conversation about a set of figure-like stimuli with spatial attributes while each of them was assigned a particular role (Director or Matcher, see "Method" section). As such, the content of their talk focused on references to concrete concepts as well as spatial information, and the exchange was structured in the sense that those participating in it adopted particular roles. However, both participants took turns at contributing their 
thoughts, ideas, and interpretations to the discourse, and they created those contributions spontaneously (in terms of both content and timing), thus fulfilling the very definition of dialogue. Further, the interaction (in the latter condition) was characterized by the ten fundamental features of face-to-face conversation as defined by Clark (1996; see also Clark and Brennan 1991).

Our main aim was to examine gestural mimicry in face-to-face dialogue by comparing the interactants' behavior here to that occurring in the absence of visibility to create a baseline for mimicry occurring by chance. Further, the task required participants to achieve a shared conceptual understanding of the stimuli, thus allowing us to focus on co-speech gesture mimicry during the collaborative creation of referring expressions, a process that is core to dialogue. Based on previous research, we predict that participants will mimic each other's co-speech gestures, and that, although some mimicry may occur due to chance, they do so significantly more frequently when they can see each other's gestures. In addition, by providing some detailed descriptive analyses, we aim to identify and illustrate some of the specific uses and functions of mimicked gestures in the dialogic process of creating a mutually shared understanding of referring expressions.

\section{Method}

\section{Design}

The experimental design was based on Clark and Wilkes-Gibbs (1986) but included a faceto-face condition in addition to the original condition in which visibility was obstructed by a wooden screen (implemented as a between-participants factor called 'visibility', with two levels: 'f-t-f' and 'screen'). The study was originally designed to test a number of different hypotheses, which is why a second manipulation was included requiring participants to carry out the same basic task over six consecutive trials (with the aim of testing whether participants gesture less if they have more mutually shared knowledge, an analysis which we will not report in the present article due to the different theoretical focus). In order to answer the present question, that is, whether participants in dialogic face-to-face interaction mimic each other's gestures, this latter manipulation is not of interest. The data will therefore be analyzed as stemming from a design involving the manipulation of visibility only (i.e., by collapsing the data across the six trials).

\section{Participants}

Thirty-two individuals from the University of Manchester (UK) population (a mixture of undergraduate and postgraduate students from a wide range of academic departments as well as administrative staff) took part in the experiment. Participants were recruited through advertisements posted across campus and emails circulated via the university announcement system. The mean age was 22.81 years in the screen condition $(\mathrm{SD}=3.82)$, and 26.50 years in the face-to-face condition $(\mathrm{SD}=6.37)$. These were randomly grouped into pairs, of which eight pairs took part in each visibility condition. The number of same-gender dyads (one male, five female) and mixed-gender dyads (two, with the female participant in the director role) were the same across both conditions. All participants were native English speakers and not acquainted with their experimental partner (other than by 'hello'). 
Apparatus and Materials

We used two identical sets of 12 geometrical figures (prints of shapes used in the ancient Chinese game of tangram), which were originally selected by Clark and Wilkes-Gibbs (1986) based on a book showing a large array of such shapes (Elffers 1976). Each figure was black printed on a white background and made into a laminated card measuring $10 \mathrm{~cm} \times 13 \mathrm{~cm}$ in size (see Fig. 1).

Each set of cards was placed onto a table $(150 \mathrm{~cm}$ wide, $75 \mathrm{~cm}$ deep, $70 \mathrm{~cm}$ high) placed opposite each other at a distance of about $3 \mathrm{~m}$. Twelve yellow squares, measuring the same size as the cards, were glued onto each table, arranged in two horizontal rows of six. On one table, the cards were placed onto the 12 squares. On the other, the cards were placed along the bottom edge of the table, in one long line. A cardboard screen, $20 \mathrm{~cm}$ in height, was affixed to the front of each table, preventing view of the cards from the respective opposite table. In the condition where visibility of the other participant was fully blocked, an additional large wooden screen (about $2 \mathrm{~m} \times 2 \mathrm{~m}$ in size) was placed midpoint between the two tables.

Two wall-mounted cameras were used to record the participants' verbal and nonverbal behavior, each providing the view of one participant and feeding into the recorded video image in a split-screen format.

\section{Procedure}

Two participants (randomly paired) took part in the experiment at a time, randomly allocated to one of the two visibility conditions. Each participant took on one of two roles, that of Director (D) or that of Matcher (M); this allocation was also random. D was asked to stand in front of the table which had the cards already placed on the squares, thus determining the individual position of each card. M stood in front of the table where the cards were placed next to each other in one long line. D was instructed to communicate to $\mathrm{M}$ where each card had to be placed, for all 12 cards. They were given as much time as they needed to complete this process and were free to talk as much about the shapes as they felt was necessary in order for M to match D's order of cards. When they thought they had achieved a full match, they were asked to notify the experimenter (who sat at the opposite end of the room, reading, with the back turned towards the participants). The experimenter noted their accuracy, reordered the cards on D's table (according to a systematically

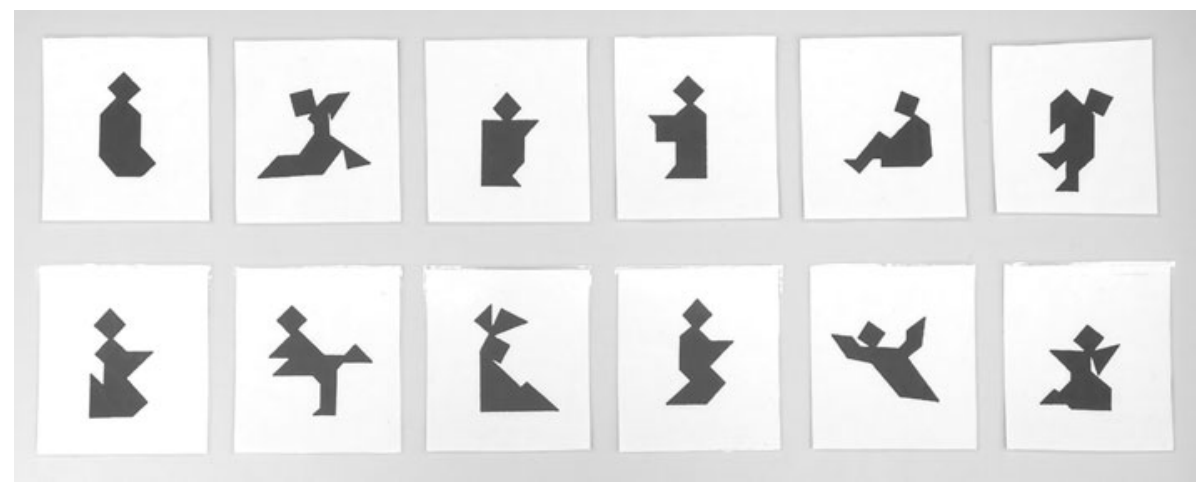

Fig. 1 Tangram cards used as task stimuli 
randomized order), shuffled the cards on M's table and placed them along the bottom edge of the table in the order in which the cards appeared in the shuffled pile. The participants were asked to return to their tables, and the same process was repeated another five times. Participants were not informed about their performance until they had completed all six trials to avoid that learning about their performance would influence their gestural behavior on the next trials (e.g., more gestures in case of negative feedback). (Despite some pairs achieving $100 \%$ of correct matches already on trial 1 , the process of creating shared conceptualizations and referring expressions evolved throughout the experiment). Also, participants were asked not to discuss anything related to the experiment in between trials. In total, each experimental session lasted for 30-45 min. At the end of each experiment, participants were fully debriefed, financially compensated ( $£ 6$ per person) and thanked for their participation.

\section{Gesture Analysis}

As a general first step, we aimed to identify all co-speech gestures accompanying references to the figures on the stimulus cards using McNeill's (1992) categories of iconic, metaphoric and deictic gestures, as well as interactive gestures (Bavelas et al. 1995). Two judges (one of them blind to the experimental hypotheses) independently categorized $17 \%$ $(N=692)$ of the gesture data using the above definitions, which resulted in a Cohen's Kappa coefficient of agreement of $K=.84$, thus indicating high reliability. However, the analysis reported here focuses on a smaller subset of these gestures (see below).

\section{Gesture Mimicry}

With the term 'mimicked gestures', we are referring to gestures highly similar in their form and in the meaning they depict. Like Scheflen (1964), we considered both carbon copies (e.g., in the context of the participants' opposite location, D and M depicting a shape that juts out to each of their respective left hand side) and mirror images (i.e., D depicting a shape that juts out to his/her left which $M$ repeats as jutting out to his/her right) in our analyses. Although a wider range of gesture types was originally identified (for other analyses to be carried out on the data and to calculate the inter-observer reliability), we focused our identification of co-speech gesture mimicry on iconic and metaphoric gestures. The rationale for excluding deictic gestures was that, due to their nature, gestures fulfilling a pointing function usually adopt a very limited range of forms anyway (e.g., pointing with the index finger extended or with the whole hand extended, and with the arm fully outstretched or not). To find that interactants use congruent forms for their pointing gestures is therefore not terribly surprising. A similar argument applies to interactive gestures, which usually involve the palm facing upwards, the hand being open and directed towards the addressee (see Bavelas et al. 1995), which were therefore also excluded from this particular analysis.

The criteria used for establishing whether a gesture should count as mimicked or not were as follows: (1) The gestures had to represent the same meaning and refer to the same entity (e.g., a gesture representing the diamond shaped head of a figure, and one representing, in the same way, the figure's diamond shaped body would not be considered mimicked; likewise, a gesture representing the diamond-shaped head of one figure in the same way as that of another figure would not be considered a mimicked gesture pair). (2) Mimicked gestures show the same 'mode of representation' (Kendon 1988; Müller 1998). For instance, if a figure's tilted head was originally represented with an iconic hand shape 
(such as the index fingers and the thumbs of both hands forming a diamond shape while the hands carry out a tilting motion to the right), then any gesture coded as mimicked would have to also involve this kind of hand movement. A re-enactment representing the figure's tilted head (such as the speaker tilting his own head to the right) would not be considered mimicked, although it represents the same meaning. As another example, an iconic gesture involving an extended index finger outlining ('drawing') a figure's long, flat, horizontal base, would not be coded as mimicked with regards to a gesture involving a flat, horizontally held, but motionless hand and lower arm representing the same feature of the figure. (3) The gestures have the same overall form. For example, if an original iconic gesture involved both hands outlining the gradual slope of a figure's shoulders, then any gesture mimicking this would have to appear similar in its overall shape/form. Thus, a onehanded gesture representing the outline of both shoulders (in quick succession and one flowing movement), would not be considered as mimicking the original gesture.

Some minor variability was accepted, however. For instance, an original gesture that held the index finger and thumb on both hands in a square shape to represent a figure's diamond head could be mimicked in a later gesture in which all four fingers and thumb on both hands represented the square shape; similarly, a gesture involving the index finger drawing a straight line was considered as mimicked if all four fingers were placed next to each other to draw the line; the rationale behind this decision was that quite a number of gestures were not easily distinguishable as one or the other as their hand shape changed during the course of the movement (e.g., the hand started off with all four fingers being held in parallel but ended with three curled in and only the index finger extended, and sometimes the opposite happened). Also, gestures did not have to be performed in the same area of the speakers' gesture space to count as mimicked providing that the change in gesture space did not change the gesture's meaning (e.g., a gestural depiction of a certain geometrical shape). The reason for this was that we applied the same criteria to the screen condition, where visibility might affect where the gesture is carried out but not the meaning it represents; thus, we stacked the odds in favor of our nil hypothesis.

Taking into account some degree of leeway in this manner allowed us to also capture cases where interlocutors did pick up on the other person's gesture but, due to increasing common ground, may have performed it in a slightly more elliptical form; that is, while the gesture may have looked more sloppy or may have been reduced by a particular semantic aspect, the general conceptualization did not change and a core aspect of the semantic representation was always retained in any gesture coded as mimicked. In some way, this resembles Kimbara's (2008) approach where gestures were coded as mimicked if they corresponded to another in terms of one out of several morphological features of the gestures (i.e., hand shape) while they may or may not have differed regarding other features (such as location, size, orientation, and movement).

In terms of the temporal distance between two gestures considered as mimicked we imposed no restrictions. Thus, all gestures that were passed between the two partners, which matched the semantic and form-based criteria mentioned above, were considered in our analysis, irrespective of the time lag (this is in line with Kimbara (2008) who identified co-referential gesture pairs contained in the entire descriptions participants produced, irrespective of their temporal distance). Further, because both partners took turns at contributing to the discourse (including the presentation of references and acceptance, or revision of these) we considered both gestures that were passed from $\mathrm{D}$ to $\mathrm{M}$ and vice versa.

Calculation of the inter-observer-reliability for identifying mimicked gestures, based on $21 \%$ of the data $(N=850)$, resulted in a Cohen's Kappa of $K=.83$, indicating high 
reliability. (Although the second coder was not informed about the experimental hypotheses to be tested, we cannot exclude the possibility that she may have inferred the analysis' aim through the obvious manipulation of visibility in the two conditions.)

For each person, the number of mimicked gestures was divided by the total number of gestures they had produced to yield the proportion of mimicked gestures. This was done to account for the fact that some participants simply gestured more than others in general and may have produced a higher number of mimicked gestures as a consequence of this, hence representing a potential confound.

\section{Mimicry Function}

In addition to coding the gestures for mimicry, we also examined them for the functions they appeared to fulfill and for their interaction with speech in this context. This process resulted in three categories that were considered to fully capture the cases of mimicry found in the present dataset. The categories were named presentation, acceptance, and displaying incremental understanding. The presentation category was loosely based on Clark and Wilkes-Gibbs' (1986) definition of the term and comprised all noun phrases as well as more complex descriptions of the stimuli (i.e., any verbal and/or gestural utterance used to refer to the intended stimulus). The acceptance category includes all those cases that, based on Clark and Wilkes-Gibbs, fulfilled the function of asserting acceptance of a presentation. The displaying incremental understanding category refers to cases where interlocutors signaled that they had understood to some degree but were still trying to figure out exactly which one the intended referent is (e.g., by indicating that they were following the other's referential information attentively and slowly [often involving a concentrated effort]), or by performing the gesture slightly more hesitantly while examining their cards after looking at their interactant. A second judge categorized $40 \%$ $(N=45)$ of the gestures mimicked in the face-to-face condition independently from the first judge; the inter-observer-reliability for categorizing the mimicked gestures according to the aforementioned criteria resulted in Cohen's Kappa of $K=.80$, again indicating strong agreement. (Regarding all reliabilities reported here, discrepancies between judges were resolved through subsequent discussion.)

\section{Results}

Gesture Mimicry in the Face-to-Face and Screen Conditions

In all, mimicked gestures were produced by seven out of the eight pairs in the face-to-face condition. The pairs in the screen condition were also examined for gestures that fulfilled our mimicry criteria to determine how often such matches would occur by chance, which was the case for five out of the eight pairs.

Shapiro-Wilk tests confirmed that the data were not normally distributed and nonparametric statistics were carried out in accordance with this. In line with our directional hypotheses, one-tailed tests were used and an alpha value of .05 .

Participants in the face-to-face condition mimicked 113 of their gestures. In the screen condition, they produced a total of 31 gestures that fulfilled the form criteria for mimicked gestures.

To account for differences in the overall amount of gestures used by different participants (and thus a potential difference in 'opportunities' to produce mimicked gestures) we 
based our statistical analyses on the proportion of mimicked gestures. ${ }^{1}$ A Mann-Whitney $U$ test showed that speakers in the face-to-face condition mimicked a significantly higher proportion of each other's gestures than speakers in the screen condition, $U=12.50$, $n_{1}=8, n_{2}=8, p=.019, r=.52$ (face-to-face: Mdn $=.04$, Range $=.11$; screen: Mdn $=.01$, Range $=.06$ ), thus confirming our hypothesis.

\section{Functions of Gesture Mimicry}

The statistical analysis reported above provides evidence that the mimicry of co-speech gestures in the face-to-face condition did not just occur by chance but must be functional in nature. This section explores some of these functions.

A qualitative examination of all instances of gesture mimicry revealed that they fall into three function categories: presentation, acceptance, and displaying incremental understanding (see "Method" section). This categorization showed that just above half of the mimicked gestures were used by speakers as part of a presentation of a noun phrase (or of a more complex description). Over a third of all mimicked gestures were classed as asserting acceptance, and a much smaller number served the signaling of what we termed incremental understanding (see Table 1). A closer examination of these gesture uses suggests that they may play an important role in the process of achieving a mutually shared understanding of referring expressions.

\section{Presentations}

Mimicked gestures were often used by speakers as part of the presentations they made involving partial or full depictions of the shapes. Here, the mimicked gestures appeared to serve a range of different functions.

Firstly, on several occasions, the mimicked gestures became closely associated with the verbal referring expression when they were passed between $\mathrm{D}$ and $\mathrm{M}$ with repeated references to the same figure. In some cases, this concerned individual components of the shapes, such as a verbal reference to a figure's straight side being accompanied with a gesture drawing a vertical straight line in the air. In other cases, mimicked gestures depicted the shape as a whole, for example, verbal references such as "there's one that looks a bit like a ghost going "woo'", or "the ghost, "woo'", each time accompanied by a gesture that imitates a ghost-like movement, involving the body moving forward/sideward, with both arms being raised. Another example is the repeated reference to a figure referred to as "the ice skater", accompanied by a re-enactment of the position of what was interpreted as a person's body and limbs. With respect to the last two examples in particular, the mimicked gestures may be considered as having become part of conceptual pacts, i.e., implicit agreements between the participants on how to conceptualize a particular entity, which is reflected in their entrainment on a particular verbal reference (Brennan and Clark 1996). In this case, the mimicked gestures seem to be an integral part of these entrained referring expressions.

However, on other occasions, the link between the gesture and the verbal part of the referring expression seemed to be more flexible in that the mimicked gesture allowed for more "sloppy" word choice (even resulting in a lack of verbal entrainment at times). For example, D and $\mathrm{M}$ had negotiated the conceptualization of a shape as something that looked like a person with two arms sticking out behind their back, which M accompanied

${ }^{1}$ Carrying out the statistical tests based on the frequencies did not change the statistical results. 
Table 1 Total number and percentages of mimicked gestures for each of the three mimicry function categories

\begin{tabular}{lcc}
\hline & Frequency & Percentage (\%) \\
\hline Presentation & 64 & 57 \\
Acceptance & 40 & 35 \\
Displaying incremental understanding & 9 & 8 \\
Total & 113 & 100 \\
\hline
\end{tabular}

with a gesture involving her two arms representing the position of the figure's arms. On a later trial, D mimics this earlier gesture, but she accompanies it with a verbal utterance that refers to "two things sticking out behind" instead. Here, the fact that her gesture is understood by both as representing the figure's arms and their position makes her utterance more precise and allows her to verbally refer to: "things" rather than "arms". Thus, when considered in isolation, the verbal utterance does not appear entrained with earlier ones; however, when conceiving of her utterance as comprising both speech and gesture, the referential continuity is still evident.

In yet other cases, through their iconic nature, the gestures appeared to anchor referring expressions in the physical world; sometimes, this allowed participants to entirely change the conceptualization of a shape. For example, D referred to a particular figure as "an ostrich" accompanied by a full body re-enactment of the figure. An attempt was made to ground this presentation, followed by $\mathrm{M}$ offering a different verbal interpretation, stating "Yeah, okay that, that looks like a woman to me, kicking her leg up behind her, yeah?", accompanied by the same gesture previously performed by D.

Thus, it appears that the mimicked gestures here functioned as part of presentations in various ways, such as by constituting an integrated whole together with the verbal utterance, resulting in both verbal and gestural alignment, or by doing away with the need of an entrained verbal utterance, using gesture to carry most of the communicational burden of providing a mutually understandable referring expression. In either case, the mimicked gestures appeared to facilitate the mutual understanding of the particular aspect that was being referred to (the entire figure or individual parts of it).

\section{Acceptance}

That mimicked gestures play a role in the process of grounding is even more apparent when considering those cases categorized as asserting acceptance. Example 1 illustrates such a case when considering both of the gestures (1 and 2). D initiates her referring expression and, half way through, as she produces a gesture as part of that reference, $\mathrm{M}$ mirrors the gesture accompanied by verbal acceptance of the presentation. Interesting is that the gesture here is a more powerful way of asserting that she has understood; the speech contains no semantic information relating to the figure at all, whereas the gesture does (it represents the two arms sticking out from the back), thus leaving D in less doubt that M has fully understood. Whereas the word "yeah", on its own, can sometimes signal continued attention and understanding "so far" (while expecting more information to come), the mimicked gesture, as used in this example, signals in a more definite manner that the entirety of the reference has been understood.

Example 2 illustrates an even clearer case of $\mathrm{M}$ employing a mimicked gesture as a powerful means of expressing acceptance. Here, the referring expression the participants create emerges from a highly collaborative process. They are referring to the respective figure for the second time and D initiates a verbal reference accompanied by a gesture 
(which was used for the first time by $\mathrm{D}$ when referring to the same figure earlier on). In response to seeing this gesture, $\mathrm{M}$ completes the verbal reference initiated by $\mathrm{D}$, resulting in a split utterance. Both, simultaneously (with just a very small time lag), produce the same gesture while looking at each other. Importantly, as D realizes that $\mathrm{M}$ has understood her gestural reference, she abandons her verbal utterance, but not her gesture, showing that the gestural presentation is clearly part of their common ground. More importantly, neither of the interactants expresses acceptance verbally during or following the referring expression-the mimicked and simultaneously performed gesture seems fully sufficient to ground the reference that they have jointly presented. It unambiguously signals what participants in other cases added verbally, such as by saying "I'm with you" or "I got you".

Example $1^{2}$

D: /Second row, number 6 is/ sitting down, [(1) with two

M: [(2) Yeah]

D: things] sticking out behind

M: Alright

Gestures (1) and (2) from this sequence are shown in Fig. 2 (D appears on the left hand side of the split screen, $M$ on the right).

Example 2

D: /The next row/ [(1) (D gestures without speaking, as M completes the turn)] M:

[(2) You've got hands up]

Gestures (1) and (2) from this sequence are shown in Fig. 3 (D appears on the left hand side of the split screen, $M$ on the right).

The above examples show that the mimicked gestures categorized as asserting acceptance occurred in different contexts. They accompanied verbal expressions of acceptance combined with a verbal referring expression (e.g., "the pointy one, yeah", accompanied by a movement of the extended index finger drawing a pointy angle in the air), or they accompanied just a verbal expression of acceptance (e.g., "yeah" accompanied by a gesture). However, in some cases, a mimicked gesture was used to assert acceptance even without any accompanying speech being produced at all, thus carrying the full burden of asserting acceptance. The fact that in $46 \%$ of cases (see Table 2) the semantic information represented in the mimicked gestures used to assert acceptance was not represented verbally at all underlines the important contribution mimicked gestures make to the grounding process.

\section{Displaying Incremental Understanding}

A smaller number of cases were involved in the grounding process by signaling incremental understanding as well as an explicit attempt made by the gesturer to create a shared understanding of meaning. Such mimicked gestures tended to be produced without speech (apart from one case where it accompanied a question). This occurred, for example, when M tried to comprehend D's presentation and match the suggested conceptualization to one of their shapes (i.e., after looking at the speaker, the gesture is mimicked while gazing at

\footnotetext{
${ }^{2}$ Dashes mark parts of speech not included in the analysis (see "Method"). Square brackets indicate the start and end points of a gesture, and numbers in parentheses included within the brackets number the gestures consecutively; underlined gestures are pairs of mimicked gestures.
} 


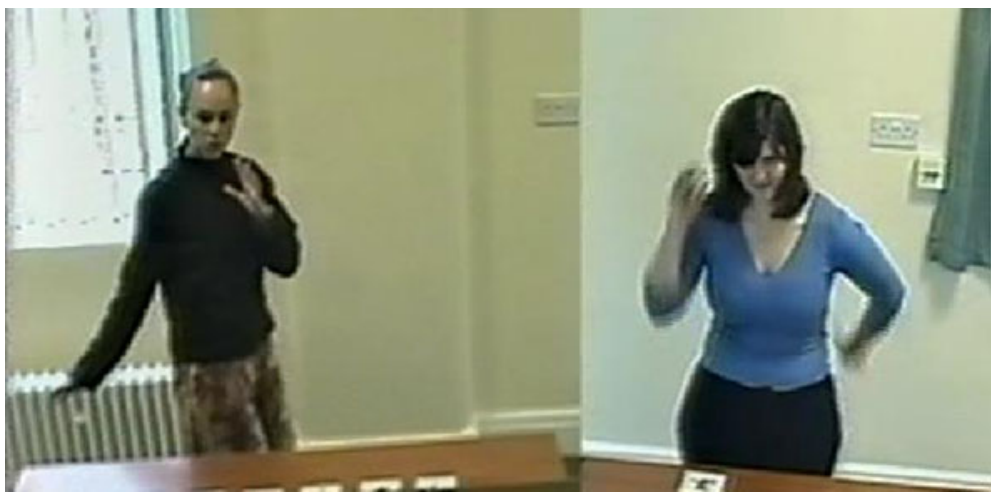

Fig. 2 Example of the Director using a mimicking a previous gesture as part of her presentation and the Matcher mimicking the Director's gesture to assert acceptance

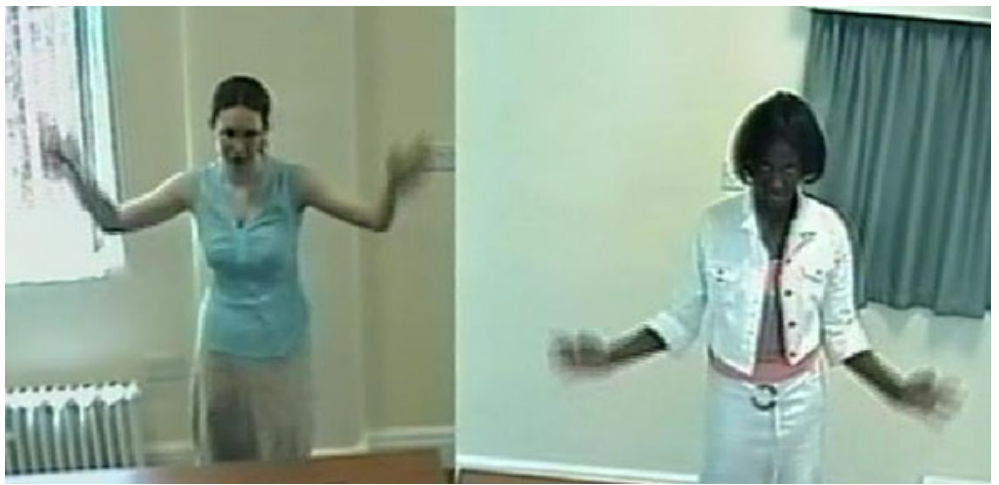

Fig. 3 Example of the Matcher mimicking the Director's gesture asserting acceptance in a split-utterance context

Table 2 Interaction of speech and mimicked gestures in the process of asserting acceptance

\begin{tabular}{lcc}
\hline Verbal modality & Frequency & Percent $(\%)$ \\
\hline Verbal expression asserting acceptance + verbal referring expression & 22 & 55 \\
Verbal expression asserting acceptance only & 15 & 38 \\
No speech at all & 3 & 8
\end{tabular}

Note that the figures displayed in this column add up to 101 due to the rounding of figures. The numbers including decimal places for the last two categories are 37.5 and 7.5

their own cards, as if to signal "I've understood what you've said but am not sure I know which figure you're referring to just yet-I might need more information or I might just need to think a bit longer about which card it could match"). In other cases, the gestures were mimicked by carrying them out at the same time as the interactant in an attempt to signal something that could be paraphrased as "I am following what you are saying in an effort to reach shared understanding with you".

Example 3 (and Fig. 4) demonstrates such a case. The participants encounter a problem in coordinating their understanding of which shape they are talking about. In this case, the 
Fig. 4 Example of the Matcher using a series of mimicked gestures in close succession to signal incremental understanding

$$
(15)+(16)
$$

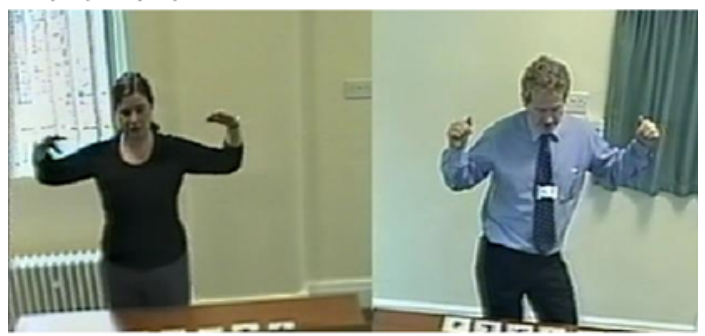

$(21)+(22)$

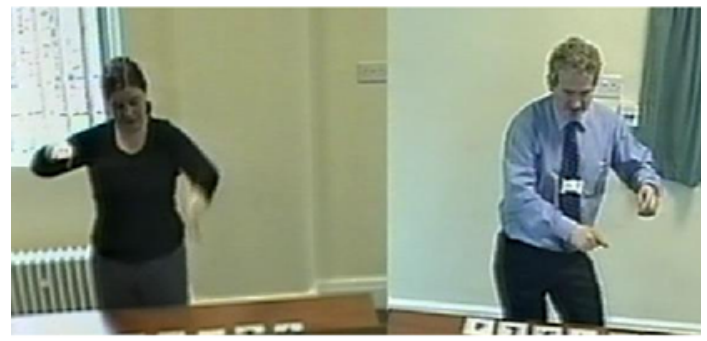

$(23)+(24)$

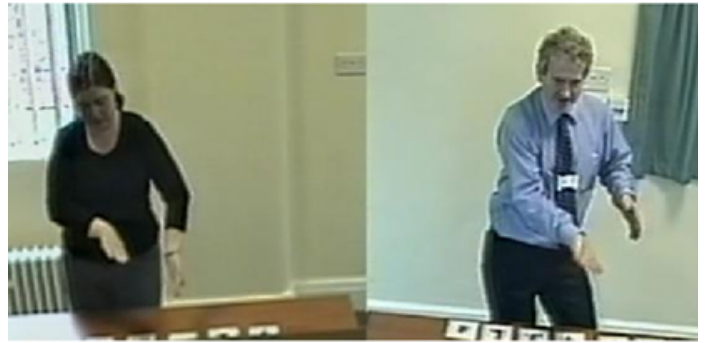

$(25)+(26)$

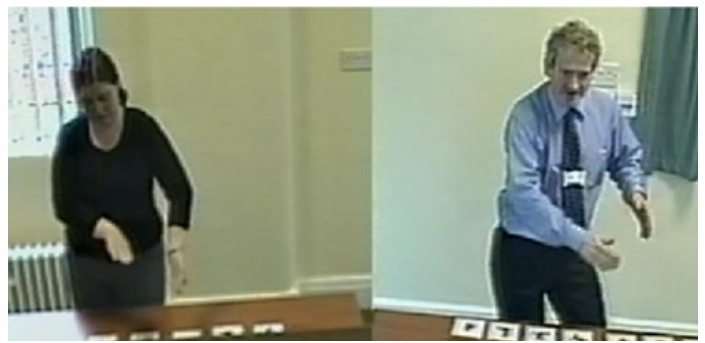

interactants make a very explicit attempt to align on their description and resulting conceptualization. They mimic several gestures in close succession, and they perform these gestures slowly and clearly oriented towards the respective other, in the centre of the gesture space. Some of the mimicked gestures are produced by $\mathrm{M}$ without him producing any speech but as accompaniments of D's verbal description and in synchrony with her gestures (e.g., (16), (20), (22)). In some sense, these carefully and synchronously performed mimicked gestures represent an on-line reflection of the current status of their 
common ground. At the same time, the extract illustrates the highly collaborative nature of the grounding process in face-to-face dialogue.

Example 3

D: /Um this next one is/ well it's, /th-/ [(1) so there's a diamond head] [(2) and then underneath there's a rectangle block] [(3) but down the right hand side it's got something cut out of it]

M: Okay

D: It's

M: So, [(4) on this one] [(5) when the head meets their shoulders] [(6) and goes off to the, the right] [(7) it extends above the rest of the body] [(8) and then comes back down into it?]

D: No

M: No, okay

D: No, [(9) it's /uh/]

M: [(10) A-are the shoulders, if you just look at the length of the shoulders]

D: [(11) The shoulders are quite broad]

M: [(12) and it]

D: [(13) The, there, there's the square on the top]

M: [(14) Yeah]

D: [(15) Shoulders are quite broad]

M: [(16) - no speech - $]$

M: [(17) And it tips]

D: [(18) One side just goes straight down]

D: [(19) and the other side sort of cuts in]

M: [(20) - no speech -]

D: [(21) goes straight down]

$\mathrm{M}:$ [(22) - no speech -]

D: [(23) cuts in]

M: [(24) in]

D: [(25) and then out, it's]

M: [(26) and slides out a bit], [(27) but yeah]

D: [(28) It's like feet at

M: I'm with you

D: the bottom]

M: We're done

Some of the pairs of mimicked gestures from this sequence are shown in Fig. 4 (again, D appears on the left hand side of the split screen, $M$ on the right - the shots are stills from the actual video, which means that $\mathrm{D}$ and $\mathrm{M}$ were performing these gestures in synchrony; however, due to a slight time lag between their gestures—D tended to be "leading" — some of the shots show small differences, e.g., when one person's wrist had already turned in preparation for the subsequent movement, but the other had not progressed in their movement to the exact same point yet).

\section{Discussion}

Our main finding is that co-speech gesture mimicry does occur in face-to-face dialogue, the form of talk we most frequently engage in. Moreover, the present study demonstrates that 
these mimicked gestures play an important role in creating mutually shared understanding. The analyses show that the mimicked co-speech gestures are both part of the common ground interactants accrue, as well as part of the very process by which they do so; the mimicked gestures appear to play an active role in the grounding process, in particular with respect to asserting acceptance (thus, it builds on previous individual observations of gesture mimicry or uptake of gesture features used to express understanding, e.g., de Fornel 1992; Holler 2003; Kimbara 2006; Streeck 2009; Tabensky 2001). Communicating acceptance is a core step in the process of reaching shared understanding in dialogue (Clark and Wilkes-Gibbs 1986). Thus, the present findings build on, and significantly advance, our understanding of co-speech gesture mimicry, which so far had been shown to occur in non-interactional contexts (Parrill and Kimbara 2006) and in the rather specialized context of co-narrations (Kimbara 2008).

While the present data are certainly not representative of all types of talk and conversational contexts, they are based on a task that requires the participants to coordinate their understanding by each contributing their ideas and interpretations - thus fulfilling the fundamental features that define dialogue. Also, the data do fulfill the ten basic characteristics of face-to-face conversation specified by Clark (1996). In addition, the stimuli we used evoke communication focusing on things that frequently feature in everyday talk, such as spatial information (which we find in route descriptions, the description of shapes of objects, their relative position with regard to one another, and so forth) as well as the names of everyday objects and their attributes (e.g., an ice skater with their arms out; a donkey with large ears sitting down). The process of referring is core to human language. The present data thus capture an important facet of communication. However, we may not necessarily be able to generalize the present findings to more unstructured types of exchange, such as conversation that does not focus so explicitly on the grounding of referring expressions. And, of course, talk of different content (e.g., about more abstract topics like political discussions, or emotions) may not necessarily encourage gesture mimicry to the same extent.

With regard to the domain of conversation examined here, our study provides evidence of co-speech gestures fulfilling important communicative functions and support the notion that they form an integral part of the collaborative process of language use (Clark 1996). Much of the previous research on gestural communication has used paradigms in which verbal interaction was seriously restricted (such as through explicit instructions to the addressee not to ask questions or through interaction with a confederate/experimenter who provided minimal feedback for the purpose of comparability-e.g., Alibali et al. 2001; Chu and Kita 2008; Holler and Wilkin 2009; Hostetter and Alibali 2007; Jacobs and Garnham 2007; Melinger and Levelt 2004; Mol et al. 2009). This underlines the importance of the dialogic focus of the present study. Here, we aimed to add to our comparatively sparse knowledge of the functions of gestures in this domain by illuminating their role in referential communication, one important aspect of dialogue.

The present study lends strong support to the notion that gestures are produced with communicative intent (Bavelas and Chovil 2000; Clark 1996; Kendon 1985). Theories of gesture production postulating a facilitatory role of gestures in lexical access or conceptual planning (Butterworth and Hadar 1989; Hostetter et al. 2007; Krauss et al. 2000) are unable to explain our findings of gesture mimicry. Of course, in general, co-speech gestures may be multi-functional, and the present study did not set out to test one gesture production theory against another. However, our findings are not agreeable with the notion that gestures are involved in the communication process in a merely trivial manner (Krauss et al. 2000); in fact, we show that they are an essential component of it. 
The finding that interlocutors pick up on each other's gestures during dialogue is further evidence that co-speech gestures do indeed communicate information to recipients, in this case information which is core to successful referential communication. A host of previous studies has measured the communicative effectiveness of gestures using paradigms in which recipients are presented with video-recorded gestures (Beattie and Shovelton 1999; Kelly and Church 1998; Riseborough 1981; Rogers 1978), or with actors who have been trained to reproduce scripted gestures in a face-to-face context (Holler et al. 2009). Our findings provide evidence that co-speech gestures are communicative also when they occur in spontaneous, face-to-face, dialogic interaction.

Further, the present study advances our knowledge of grounding, a fundamental prerequisite for mutual understanding and coordination in interaction. A substantial amount of research has been dedicated to investigating this domain. However, the majority of this research has focused on verbal communication alone (e.g., Brennan and Clark 1996; Clark and Wilkes-Gibbs 1986; Fussell and Krauss 1992; Isaacs and Clark 1987). The interlocutors in these studies tended to be separated by an opaque screen but, apart from this, were free to communicate naturally. While such a paradigm preserves dialogic interaction (thus creating a situation similar to a telephone conversation), it prevents the interlocutors from communicating gesturally. The present findings therefore contribute to expanding research on coordination in dialogue to face-to-face communication. In particular, it illuminates in detail some of the uses of co-speech gestures that appear to facilitate this coordination.

One thing to bear in mind is that grounding is an incremental process, and one that helps to update interactants' common ground on a moment-by-moment basis (Clark and Brennan 1991). The instances of mimicry constituting the present dataset appear to facilitate grounding with regard to the particular aspect of the referring expression that they relate to. However, it needs to be clear that, from this sort of data, we are not able to argue that, on the whole, communication must have been more successful in the face-to-face context than when vision was obscured. This is because the mimicked gestures often represented only one element of a figure, and a lot of verbal and gestural information was provided preceding, or subsequent to, the respective mimicked gesture (especially on the initial trials, where most of the mimicry occurred). These other parts of the referring expressions could have been misunderstood, thus preventing successful identification of the figure as a whole. Furthermore, grounding refers to the participants' belief of having achieved a shared understanding of their partner's contribution-for example, on the basis of a mimicked gesture. However, this did not always match their actual accuracy (e.g., because they miscommunicated on some preceding or following aspect of the referring expression). This means that, although mimicked gestures appeared to benefit the grounding of the particular semantic aspects they represented, this did not necessarily guarantee successful communication of the more complex utterance constructs (consisting of several phrases or sentences) in which they were embedded. Because the accuracy data we recorded showed that participants performed at ceiling level (in both conditions), we cannot claim that communication in the face-to-face condition was better overall, and that this would have been partly attributable to the mimicked gestures in this condition. However, our focus here is on the use of co-speech gesture in collaborative, dialogic interaction processes. For the current purpose, we therefore consider the more meaningful analysis to be one that focuses on the direct benefit of the mimicry for the grounding process, that is, on the immediate communicative and interactive context, as illustrated in our examples. Through this microfocus, the present analysis complements existing research on gesture and common ground (Gerwing and Bavelas 2004; Holler and Stevens 2007; Holler and Wilkin 2009; Parrill 
2010) as it provides us with an insight into some of the functions of gestures that help interactants to update common ground on a moment-by-moment basis.

So far in our discussion (and in our analysis) we have considered gestures as functional in dialogic interaction due to the semantic content they convey. Of course, co-speech gesture mimicry may have social-affective functions, too. There is ample evidence that mimicry regarding other nonverbal behaviors, such as posture or self-adapters, is significantly correlated with interpersonal rapport and liking. Based on our dataset, we cannot, and do not aim to, exclude the possibility that co-speech gesture mimicry has similar effects. However, what we would argue is that any such effect would exist in addition to the semantic and communicative functions we have mapped out here. Future research may throw more light on this issue.

Another important question is the degree of intentionality/automaticity associated with the mimicked gestures. Researchers argue that behavioral mimicry (both verbal and nonverbal) is a nonconscious, unintended process, based on an automatic priming mechanism involving a perception-behavior link (e.g., Dijksterhuis and Bargh 2001; Pickering and Garrod 2004). While we cannot firmly rule out this interpretation regarding the mimicked behavior observed in the present dataset, we would argue that the majority of the mimicked gestures were used in a context that makes an intentional, conscious use a more plausible explanation. As illustrated through the examples in our "Results" section, in many cases, the gestures carried much of the communicative burden (e.g., when accompanying single words confirming understanding), or were part of a concentrated effort to signal understanding (or the attempt to reach it), often combined with being the only modality used to convey the respective semantic information. Lakin and Chartrand (2003) differentiate between conscious and nonconscious mimicry and point out that, depending on the type of nonverbal behavior under investigation, one may observe one or the other. They state that what they call the "passive, simple behaviors" which they observed (such as foot shaking and face touching) are associated with nonconscious mimicry. Co-speech gestures, which are part of the actual communicative message one conveys, may be one type of nonverbal behavior associated with a more conscious mimicry process, at least in the context of referential communication which we have focused on here. Further research is needed to uncover the exact mechanisms underlying mimicry in co-speech gesture.

To conclude, the present study has provided clear evidence that interlocutors in face-toface dialogue mimic each other's co-speech gestures, and that this sort of mimicry is anything but accidental. Furthermore, we have attempted to identify some key functions of these mimicked gestures which show that they are directly involved in establishing mutually shared understanding in face-to-face dialogue. Avenues for future research may comprise an investigation of the underlying cognitive mechanisms, as well as of the more social-emotional effects of mimicked co-speech gestures.

Acknowledgments We would like to express our gratitude to two anonymous reviewers, as well as action editor Professor Judith Hall, for their invaluable comments on earlier drafts of this manuscript. We would also like to thank the Economic and Social Research Council (research grant RES-061-23-0135) and the British Academy (research grant SG-48338) for funding this research. Finally, we would like to thank Rachel Poorhady for help with the data collection, as well as the participants who took part in our study.

\section{References}

Alibali, M. W., Heath, D. C., \& Myers, H. J. (2001). Effects of visibility between speaker and listener on gesture production: Some gestures are meant to be seen. Journal of Memory and Language, 44, $169-188$. 
Bavelas, J. B. (1994). Gestures as part of speech: Methodological implications. Research on Language and Social Interaction, 27(3), 201-221.

Bavelas, J. B., \& Chovil, N. (2000). Visible acts of meaning: An integrated message model of language in face-to-face dialogue. Journal of Language and Social Psychology, 19(2), 163-194.

Bavelas, J. B., Chovil, N., Coates, L., \& Roe, L. (1995). Gestures specialized for dialogue. Personality and Social Psychology Bulletin, 21(4), 394-405.

Bavelas, J. B., Chovil, N., Lawrie, D. A., \& Wade, A. (1992). Interactive gestures. Discourse Processes, 15(4), 469-489.

Bavelas, J. B., Gerwing, J., Sutton, C., \& Prevost, D. (2008). Gesturing on the telephone: Independent effects of dialogue and visibility. Journal of Memory and Language, 58(2), 495-520.

Bavelas, J. B., Kenwood, C., Johnson, T., \& Phillips, B. (2002). An experimental study of when and how speakers use gestures to communicate. Gesture, 2(1), 1-17.

Beattie, G., \& Shovelton, H. (1999). Mapping the range of information contained in the iconic hand gestures that accompany spontaneous speech. Journal of Language and Social Psychology, 18(4), 438-462.

Brennan, S. E., \& Clark, H. H. (1996). Conceptual pacts and lexical choice in conversation. Journal of Experimental Psychology. Learning, Memory, and Cognition, 22(6), 1482-1493.

Butterworth, B., \& Hadar, U. (1989). Gesture, speech, and computational stages: A reply to McNeill. Psychological Review, 96(1), 168-174.

Chartrand, T. L., \& Bargh, J. A. (1999). The chameleon effect: The perception-behavior link and social interaction. Journal of Personality and Social Psychology, 76(6), 893-910.

Chartrand, T. L., Maddux, W. W., \& Lakin, J. L. (2005). Beyond the perception-behavior link: The ubiquitous utility and motivational moderators of nonconscious mimicry. In H. J. Uleman \& J. A. Bargh (Eds.), The new unconscious (pp. 334-361). New York: Oxford University Press.

Chawla, P., \& Krauss, R. M. (1994). Gesture and speech in spontaneous and rehearsed narratives. Journal of Experimental Social Psychology, 30(6), 580-601.

Chu, M., \& Kita, S. (2008). Spontaneous gestures during mental rotation tasks: Insights into the microdevelopment of the motor strategy. Journal of Experimental Psychology: General, 137(4), 706-723.

Clark, H. H. (1996). Using language. Cambridge: Cambridge University Press.

Clark, H. H., \& Brennan, S. E. (1991). Grounding in communication. In L. B. Resnick, J. M. Levine, \& S. D. Teasley (Eds.), Perspectives on socially shared cognition (pp. 127-149). Washington, DC: American Psychological Association.

Clark, H. H., \& Schaefer, E. F. (1987). Concealing one's meaning from overhearers. Journal of Memory and Language, 26(2), 209-225.

Clark, H. H., \& Schaefer, E. F. (1989). Contributing to discourse. Cognitive Science: A Multidisciplinary Journal, 13(2), 259-294.

Clark, H. H., \& Wilkes-Gibbs, D. (1986). Referring as a collaborative process. Cognition, 22(1), 1-39.

de Fornel, M. (1992). The return gesture: Some remarks on context, inference, and iconic gesture. In P. Auer \& A. Di Luzio (Eds.), The contexualisation of language. Amsterdam: John Benjamins.

Dijksterhuis, A., \& Bargh, J. A. (2001). The perception-behavior expressway: Automatic effects of social perception on social behavior. In M. P. Zanna (Ed.), Advances in experimental social psychology (Vol. 33, pp. 1-40). San Diego: Academic Press.

Elffers, J. (1976). Tangram. The ancient Chinese shapes game. New York: McGraw-Hill.

Emmorey, K., \& Casey, S. (2001). Gesture, thought, and spatial language. Gesture, 1, 35-50.

Furuyama, N. (2002). Prolegomena of a theory of between-person coordination of speech and gesture. International Journal of Human-Computer Studies, 57(4), 347-374.

Fussell, S. R., \& Krauss, R. M. (1992). Coordination of knowledge in communication: Effects of speakers' assumptions about what others know. Journal of Personality and Social Psychology, 62(3), 378-391.

Gerwing, J., \& Bavelas, J. B. (2004). Linguistic influences on gesture's form. Gesture, 4(2), 157-195.

Goodwin, C. (1986). Gesture as a resource for the organization of mutual orientation. Semiotica, 62(1-2), $29-49$

Goodwin, C. (2000). Action and embodiment within situated human interaction. Journal of Pragmatics, 32(10), 1489-1522.

Graham, J. A., \& Argyle, M. (1975). A cross-cultural study of the communication of extra-verbal meaning by gestures. International Journal of Psychology, 10, 57-67.

Gullberg, M. (2006). Handling discourse: Gestures, reference tracking, and communication strategies in early L2. Language Learning, 56(1), 155-196.

Heath, C. C. (1992). Gesture's discrete tasks: Multiple relevancies in visual conduct in the contextualization of language. In P. Auer \& A. di Luzio (Eds.), The contextualization of language (pp. 102-127). Amsterdam: Benjamins. 
Holler, J. (2003). Semantic and pragmatic aspects of representational gestures: Towards a unified model of communication in talk. Unpublished doctoral dissertation, University of Manchester, Manchester, UK.

Holler, J., \& Beattie, G. (2003). Pragmatic aspects of representational gestures: Do speakers use them to clarify verbal ambiguity for the listener? Gesture, 3(2), 127-154.

Holler, J., Shovelton, H., \& Beattie, G. (2009). Do iconic hand gestures really contribute to the communication of semantic information in a face-to-face context? Journal of Nonverbal Behavior, 33(2), 73-88.

Holler, J., \& Stevens, R. (2007). The effect of common ground on how speakers use gesture and speech to represent size information. Journal of Language and Social Psychology, 26(1), 4-27.

Holler, J., \& Wilkin, K. (2009). Communicating common ground: How mutually shared knowledge influences speech and gesture in a narrative task. Language and Cognitive Processes, 24(2), 267-289.

Hostetter, A. B., \& Alibali, M. W. (2007). Raise your hand if you're spatial: Relations between verbal and spatial skills and gesture production. Gesture, 7, 73-95.

Hostetter, A. B., Alibali, M. W., \& Kita, S. (2007). I see it in my hands' eye: Representational gestures reflect conceptual demands. Language and Cognitive Processes, 22(3), 313-336.

Isaacs, E. A., \& Clark, H. H. (1987). References in conversation between experts and novices. Journal of Experimental Psychology: General, 116(1), 26-37.

Jacobs, N., \& Garnham, A. (2007). The role of conversational hand gestures in a narrative task. Journal of Memory and Language, 56(2), 291-303.

Kelly, S. D., \& Church, R. (1998). A comparison between children's and adults' ability to detect children's representational gestures. Child Development, 69, 85-93.

Kendon, A. (1980). Gesticulation and speech: Two aspects of the process of utterance. In M. R. Key (Ed.), Relationships of the verbal and nonverbal communication (pp. 207-228). The Hague, Netherlands: Mouton.

Kendon, A. (1983). Gesture and speech: How they interact. In J. M. Weimann \& R. P. Harrison (Eds.), Nonverbal interaction (pp. 13-45). Beverley Hills, CA: Sage.

Kendon, A. (1985). Some uses of gesture. In D. Tannen \& M. Saville-Troike (Eds.), Perspectives on silence (pp. 215-234). Norwood, NY: Ablex.

Kendon, A. (1988). Sign languages of aboriginal Australians. Cambridge: Cambridge University Press.

Kendon, A. (2000). Language and gesture: Unity or duality. In D. McNeill (Ed.), Language and gesture (pp. 47-63). New York: Cambridge University Press.

Kendon, A. (2004). Gesture: Visible action as utterance. Cambridge: Cambridge University Press.

Kimbara, I. (2006). On gestural mimicry. Gesture, 6(1), 39-61.

Kimbara, I. (2008). Gesture form convergence in joint description. Journal of Nonverbal Behavior, 32(2), $123-131$.

Kita, S., \& Davies, T. S. (2009). Competing conceptual representations trigger co-speech representational gestures. Language and Cognitive Processes, 24(5), 761-775.

Krauss, R. M., Chen, Y., \& Gottesman, R. (2000). Lexical gestures and lexical access: A process model. In D. McNeill (Ed.), Language and gesture (pp. 261-283). New York: Cambridge University Press.

LaFrance, M. (1979). Nonverbal synchrony and rapport: Analysis by the cross-lag panel technique. Social Psychology Quarterly, 42, 66-70.

LaFrance, M. (1985). Postural mirroring and intergroup relations. Personality and Social Psychology Bulletin, 11, 207-217.

Lakin, J. L., \& Chartrand, T. L. (2003). Using nonconscious behavioral mimicry to create affiliation and rapport. Psychological Science, 14(4), 334-339.

Lakin, J. L., Chartrand, T. L., \& Arkin, R. M. (2008). I am too just like you: Nonconscious mimicry as an automatic behavioral response to social exclusion. Psychological Science, 19(8), 816-822.

Martin, P., \& Bateson, P. (1994). Measuring behaviour-An introductory guide (2nd ed.). Cambridge: Cambridge University Press.

McNeill, D. (1985). So you think gestures are nonverbal? Psychological Review, 92, 350-371.

McNeill, D. (1992). Hand and mind. Chicago: The University of Chicago Press.

Melinger, A., \& Kita, S. (2007). Conceptualisation load triggers gesture production. Language and Cognitive Processes, 22(4), 473-500.

Melinger, A., \& Levelt, W. J. (2004). Gesture and the communicative intention of the speaker. Gesture, 4(2), $119-141$.

Mol, L., Krahmer, E., Maes, A., \& Swerts, M. (2009). The communicative import of gestures: Evidence from a comparative analysis of human-human and human-machine interactions. Gesture, 9(1), 97-126.

Morrel-Samuels, P., \& Krauss, R. M. (1992). Word familiarity predicts temporal asynchrony of hand gestures and speech. Journal of Experimental Psychology. Learning, Memory, and Cognition, 18(3), 615-622. 
Müller, N. (1998). Transfer in bilingual first language acquisition. Bilingualism: Language and Cognition, 1, 151-192.

Özyürek, A. (2002). Do speakers design their cospeech gestures for their addressees? The effects of addressee location on representational gestures. Journal of Memory and Language, 46, 688-704.

Parrill, F. (2010). The hands are part of the package: Gesture, common ground, and information packaging. In J. Newman \& S. Rice (Eds.), Empirical and experimental methods in cognitivelfunctional research. Stanford: CSLI.

Parrill, F., \& Kimbara, I. (2006). Seeing and hearing double: The influence of mimicry in speech and gesture and observers. Journal of Nonverbal Behavior, 30(4), 157-166.

Pickering, M. J., \& Garrod, S. (2004). Toward a mechanistic psychology of dialogue. Behavioural and Brain Sciences, 27, 1-57.

Pine, K., Bird, H., \& Kirk, E. (2007). The effects of prohibiting gestures on children's lexical retrieval ability. Developmental Science, 10, 747-754.

Riseborough, M. G. (1981). Physiographic gestures as decoding facilitators: Three experiments exploring a neglected facet of communication. Journal of Nonverbal Behavior, 5(3), 172-183.

Rogers, W. T. (1978). The contribution of kinesic illustrators toward the comprehension of verbal behaviors within utterances. Human Communication Research, 5, 54-62.

Scheflen, A. (1964). The significance of postures in communicative systems. Psychiatry, 27, 316-333.

Stalnaker, R. C. (1978). Assertion. In P. Cole (Ed.), Syntax and semantics (Vol. 9, pp. 315-322). New York: Academic Press.

Streeck, J. (1994). Gesture as communication II: The audience as co-author. Research on Language and Social Interaction, 27(3), 239-267.

Streeck, J. (2009). Gesturecraft: The manu-facture of meaning. Amsterdam: John Benjamins Publishing Company.

Tabensky, A. (2001). Gesture and speech rephrasings in conversation. Gesture, 1(2), 213-235. 